\title{
Gear Fault Diagnosis in Variable Speed Condition Based on Multiscale Chirplet Path Pursuit and Linear Canonical Transform
}

\author{
Xu Shuiqing $\mathbb{D},,^{1,2}$ Zhang Ke $\mathbb{D},^{2}$ Chai Yi, $^{2}$ He Yigang, ${ }^{1}$ and Feng $\mathrm{Li}\left(\mathbb{D}{ }^{2}\right.$ \\ ${ }^{1}$ College of Electrical Engineering and Automation, Hefei University of Technology, Hefei 230009, China \\ ${ }^{2}$ College of Automation, Chongqing University, Chongqing 400044, China
}

Correspondence should be addressed to Zhang Ke; smeta@163.com

Received 8 November 2017; Revised 15 January 2018; Accepted 7 February 2018; Published 11 March 2018

Academic Editor: Chen Lu

Copyright (C) $2018 \mathrm{Xu}$ Shuiqing et al. This is an open access article distributed under the Creative Commons Attribution License, which permits unrestricted use, distribution, and reproduction in any medium, provided the original work is properly cited.

\begin{abstract}
The vibration signals analysis is a very effective and reliable method for detecting the gear failures. Because the vibration signals acquired from the gear in the variable speed condition often contain more useful fault information, the analysis of the gear vibration signals during the variable speed condition has been a hot research topic. In this paper, a method based on the multiscale chirplet path pursuit (MSCPP) and the linear canonical transform (LCT) has been applied to diagnose the gear fault in the variable speed condition for the first time. First, by using the MSCPP method to estimate the instantaneous meshing frequency, the suitable signal segment approximation to the acceleration or deceleration process can be selected. Then, because the LCT is a novel and efficient nonstationary signals analysis tool, the optimal LCT spectrum of the selected signal has been attainted to diagnose the gear faults based on the properties of the LCT. In addition, the simulations and the experimental evaluation are provided to verify the effectiveness of the proposed method.
\end{abstract}

\section{Introduction}

The gear is an important device and has a wide range of applications in industry. However, owing to abrasion and other reasons, the gears may have many kinds of faults, such as pitting, chipping, and the serious crack [1-5]. When the gear causes a local fault, the amplitude and phase of the vibration signal of the gear are modulated $[2,3,6,7]$. So the vibration signals obtained from the gear can reflect the gear's state very well. The vibration signals analysis also has been the extremely effective and reliable method for detecting the gear failures [8-11]. Meanwhile, on the one hand, the gear vibration signals obtained from the variable speed condition, that is, the acceleration or deceleration processes, often can contain more fault information compared to the stationary processes, which can detect the gear faults earlier. On the other hand, the gear vibration signals obtained from the acceleration or deceleration processes are nonstationary signals, which have low signal-to-noise ratio (SNR) in practice [11-14], which make it difficult to obtain the gear vibration signals' fault features. Hence, the diagnosis of gear faults by analyzing the gear vibration signals obtained from the variable speed condition has been a hot research topic recently.

A number of failure diagnosis methods have been used to diagnose the gear faults in variable speed condition, for example, the traditional time frequency analysis methods, self-adaptive signal processing methods, and data driven methods $[4-12,15-25]$. The traditional time frequency analysis methods, such as the short time Fourier transform [14], the Wigner-Ville distribution [10, 12], and the wavelet transform [2], will result in spectral aliasing, cross term interference, and low resolution because the vibration signals obtained from the fault gears in practice are nonstationary with low SNR [4]. The self-adaptive signal processing methods, for example, the empirical mode decomposition [5] and the local mean decomposition [17], will lead to over envelope, end effects, and distorted components, respectively. In addition, the data driven methods, such as the Elman neural network [24] and support vector machine [22], require a lot of data 
for training and classification. To solve these problems, some other data driven methods have been introduced, such as the self-organizing maps (SOM) method [26-28]. In [26], the self-organizing feature map neural network has been used to diagnose the faults of the wind turbine's converter. In [27], the SOM and minimum quantization error (MQE) method have been selected to achieve degradation assessment and fault localization. In [28], an intelligent approach based on the SOM method has been presented to machine component health prognostics. Although the SOM method does not need a lot of data, it still needs some a priori knowledge. Therefore, the diagnosis of gear faults in acceleration and deceleration processes remains an open research field and new signal analysis tools for nonstationary vibration signals are needed.

The linear canonical transform (LCT) is a generalization of the Fourier transform (FT) and the fractional Fourier transform (FRFT), which has four-parameter family of linear integral transform $[29,30]$. It performs an affine mapping of the time frequency distribution of the signal because it has additional degrees of freedom $[31,32]$. So, the LCT is more flexible and suitable for processing nonstationary signals, particularly in the linear frequency modulated (LFM) signals [33-37]. Simultaneously, the gear vibration signals obtained from the variable speed condition can be approximated to the multicomponent LFM signals within a short period of time. From the above analysis, because the vibration signals attained from the variable speed condition are nonstationary and the LCT has advantages in nonstationary signals processing, it is therefore worthwhile to explore the diagnosis of gear faults by using the LCT.

However, in variable speed condition, the gear vibration signals contain many different components, which are not approximated to the LFM signals. The vibration signals acquired from the acceleration or deceleration processes of the gears are often a short period of the gear vibration signals in variable speed condition. In order to apply the LCT method to diagnosis of the gear faults in variable speed condition, the vibration signals acquired from the acceleration or deceleration processes should be selected at first. In practice, it is difficult to only obtain the gear vibration signals during acceleration or deceleration processes directly. Nevertheless, the shaft rotational frequency (SRF) is time varying in the acceleration or deceleration processes, which can be seen as an indicator to select the acceleration or deceleration processes [1]. Hence, we can obtain the acceleration or deceleration time by estimating the instantaneous frequency (IF) of the gear vibration signals in variable speed condition. In addition, the optimal LCT parameters of the LFM signals are also determined by gradient of the IF [30]. For these reasons, we need to estimate the IF of the gear vibration signals in variable speed condition at first.

In this paper, the multiscale chirplet path pursuit (MSCPP) has been used in the estimation of the IF of the gear vibration signals, which is a widely used and efficient method for IF estimation [38]. The MSCPP method divides the time length of the analysis signal into a series of dynamic time support areas in binary form, finding atom with the largest correlation in each dynamic support region from the defined chirplet atom library, and the selected chirplet

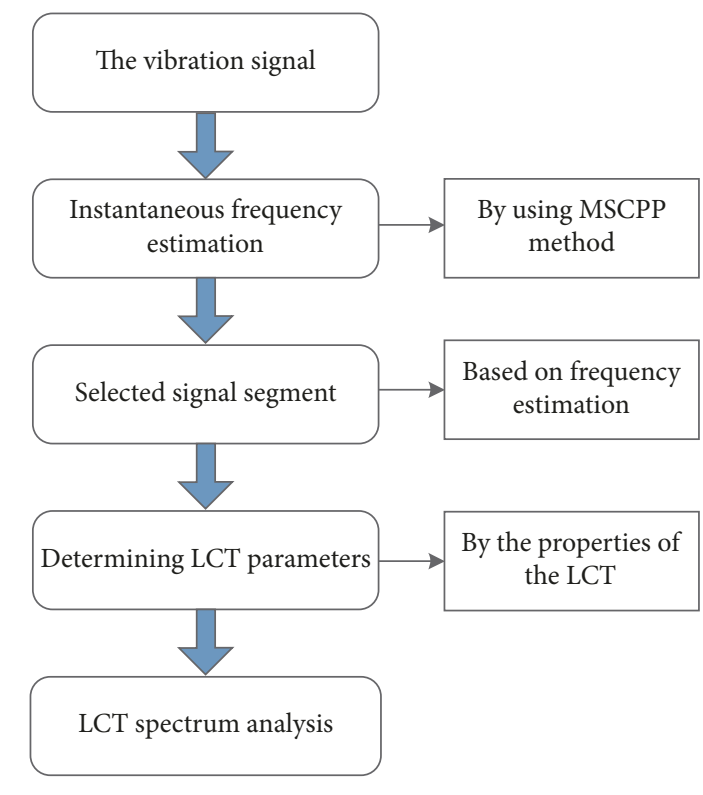

FIGURE 1: The block diagram of the proposed method.

atoms are connected one by one by using the principle of the best connection $[38,39]$. Thus, it can adaptively find the instantaneous frequency trend line with the largest correlation in the signal [39]. Compared with other instantaneous frequency methods, the MSCPP method can choose shorter chirplet atoms flexibly and can effectively suppress noise interference. In addition, the MSCPP method does not have any a priori knowledge. So the instantaneous frequency of the vibration signals obtained from the variable speed condition can be estimated by the MSCPP method. Based on this, the vibration signals obtained from the acceleration or deceleration processes in a short period of time can be picked out.

In this paper, the MSCPP method and the LCT have been applied to analyze the vibration signals to diagnose the gear faults in variable speed condition for the first time. First, by using the MSCPP, the IF of the vibration signals obtained from the variable speed condition can be estimated. Then, based on the IF of the vibration signals, we can choose the suitable signal segment, which can be seen as the acceleration or deceleration processes. In addition, according to the gradient of the instantaneous frequency of the selected signal segment, the optimal LCT parameters can be obtained based on the properties of the LCT [30]. Finally, the optimal LCT spectrum of the selected signal is presented, which can be used to diagnose the gear fault. The block diagram of the proposed method is presented in Figure 1 vividly.

The remaining parts of this article are organized as follows. In Section 2, the preliminaries of the MSCPP and the LCT are introduced. In Section 3, the application of the MSCPP and the LCT to the simulated vibration signal is presented, which shows that the proposed method is effective. The experimental evaluations have been provided in Section 4. Section 5 concludes this paper. 


\section{Preliminaries}

2.1. The Multiscale Chirplet Path Pursuit Method. The MSCPP method was first introduced in [38]; in this method, if the IF of chirplet atoms is linear, they can be used to adaptively piecewise approximate the nonstationary signals. For example, the nonstationary signal in the following is considered.

$$
y(t)=A \cos [w(t)+\varphi]+n(t),
$$

where $A, \varphi, n(t)$, and $w(t)$ represent the amplitude, the initial phase, the noise, and the successive and derivable instantaneous phase of the signal, respectively. In this algorithm, the chirplet atoms are obtained from the chirplet dictionary that can be written as a set of functions as follows [38].

$$
f_{m, n, I}(t)=|I|^{-1 / 2} e^{i\left(m t^{2} / 2+n t\right)} L_{I}(t),
$$

where $m$ is the slope coefficient and $n$ is the offset coefficient. According to (2), we can easily have that the IF of a chirplet is linear and equal to $m t+n$. Based on the sampling theorem, we know that $m t+n$ should be less than a half of the sampling rate $f_{s} . I$ is the dyadic time span, which has the binary scale division and can be defined as $I=\left\{I_{j, k} \mid j=0,1, \ldots, \log _{2} N-\right.$ $\left.1, k=0,1, \ldots, 2^{j-1}\right\}$. In this equation, $I_{j, k}=\left[k 2^{-j} T,(k+\right.$ 1) $\left.2^{-j} T\right]$. $T$ and $N$ are the total sampling time and the number of samples, respectively. $j$ is the scale coefficients and $j=$ $0,1, \ldots, \log _{2} N-1, k=0,1, \ldots, 2^{j-1} . L_{I}(t)$ is a rectangular window function, which is 1 when $t \in I$ and 0 when $t \notin I$. $|I|^{-1 / 2}$ is the normalization factor that makes $\left\|f_{m, n, I}(t)\right\|_{2}=1$.

In each time interval, by computing the maximum projection coefficient $C_{I_{j, k}}$, where $C_{I_{j, k}}$ equals $C_{I_{j, k}}=\max \langle x$, $\left.f_{m, n, I_{j, k}}(t)\right\rangle(\langle\cdot\rangle$ stands for the interior product) and includes the amplitude and initial phase information of the signal component in the time interval $I_{j, k}$, the chirplet atom which has the highest correlation to signal $y(t)$ can be obtained from the chirplet dictionary. Suppose $S_{I_{j, k}}$ is the signal component expressed by $C_{I_{j, k}}$ in the time interval $I_{j, k}$, then $S_{I_{j, k}}$ can be written in the following [38]:

$$
S_{I_{j, k}}(t)=\left|C_{I_{j, k}}\right| e^{-i\left(m t^{2}+n t\right)+\angle C_{I_{j, k}}} I_{j, k}
$$

2.2. The Linear Canonical Transform. The LCT of a signal $f(t)$ with parameter $A=(a, b, c, d)$, represented as $L_{f}^{A}(u)$, is defined by [29]

$$
L_{f}^{A}(u)= \begin{cases}\int_{-\infty}^{\infty} f(t) K_{A}(u, t) d t & b \neq 0 \\ \sqrt{d} e^{j\left(c d u^{2} / 2\right)} f(d u) & b=0,\end{cases}
$$

where $a, b, c, d$ are real numbers satisfying $a d-b c=1$. In addition, the kernel $K_{A}(u, t)$ is given by

$$
K_{A}(u, t)=C_{A} e^{j\left((a / 2 b) t^{2}-u t / b+(d / 2 b) u^{2}\right)}
$$

and $C_{A}=\sqrt{1 / j 2 \pi b}$. According to (4), it can easily be obtained that the LCT has three degrees of freedom and when the
LCT parameters are certain, the kernel $K_{A}(u, t)$ of the LCT is a LFM signal; thus the LCT of a LFM signal with suitable parameters can be a dirac function [31]. Therefore, the LCT is more flexible and suitable for processing nonstationary signals, especially for the LFM signals.

\section{The Proposed Method of the Simulated Vibration Signal}

When the gear causes a partial failure, the amplitude and phase of the vibration signal of the gear are modulated, which are periodic with the gear's rotation frequency [1]. The vibration signal $x(t)$ attained from a pair of meshing gears with tooth fault could be represented as [1]

$$
\begin{aligned}
x(t)= & \sum_{m=0}^{M} X_{m}\left(1+a_{m}(t)\right) \cos \left(2 \pi m k f_{s} t+\phi_{m}\right) \\
= & \sum_{m=0}^{M} X_{m}\left[1+\sum_{n=0}^{N} A_{m n} \cos \left(2 \pi n f_{s} t+\sigma_{m n}\right)\right] \\
& \times \cos \left(2 \pi m k f_{s} t+\phi_{m}\right),
\end{aligned}
$$

where $a_{m}(t)$ is the amplitude modulating function and $M$ represents the amount of tooth meshing harmonics. $X_{m}$ and $\phi_{m}$ indicate the $m$ th meshing harmonic's amplitude and phase, separately [1]. $k f_{s}$ is the meshing frequency, $k$ expresses the gear teeth's amount, and $f_{s}$ stands for the SRF [1]. $N$ represents sidebands' amount around the tooth meshing harmonics. $A_{m n}$ and $\sigma_{m n}$ are the amplitudes and phases at the $n$th sidebands of the amplitude modulated signals around the $m$ th meshing harmonic, separately $[2,4]$.

The SRF $f_{s}$ in (6) is time varying and the vibration signal can be approximated to the LFM signal when the instantaneous rotational speed is ascending and descending straight in the acceleration and deceleration, respectively. To verify the proposed algorithm is effective for the vibration signals; next, a signal is considered to simulate the vibration signal obtained from the acceleration process as follows based on (6):

$$
x(t)=\left[1+\cos \left(2 \pi f_{m} t\right)\right] \cos \left(2 \pi \cdot 10 \cdot f_{m} t\right)+\varphi(t),
$$

where $f_{m}$ stands for the SRF in the simulated vibration signal, which is chosen as $f_{m}=2 t+30$ when $0 \leq t<1.5$ and equal to 33 when $1.5 \leq t \leq 2$. The sampling frequency is $2000 \mathrm{~Hz}$ and the number of the sample points is set as 4096. The time interval is set as $0 \mathrm{~s}$ to $2 \mathrm{~s}$. Moreover, because the vibration signals attained from the gear in variable speed condition are usually with low SNR, $\varphi(t)$ is selected as the Gaussian white noise with the SNR is $-4 \mathrm{~dB}$. Based on the above, Figure 2 depicts the signal shown in (7) without the noise being depicted in it. The simulated vibration signal based on (7) is presented in Figure 3. Figure 4 shows the FT spectrum of the simulated vibration signal. According to Figure 3, because the signal contains noise, any useful information can not be obtained directly. Analogously, since the simulated vibration signal is nonstationary and has noise, this leads to the FT spectrum in Figure 4 that has spectral aliasing and blurring. 


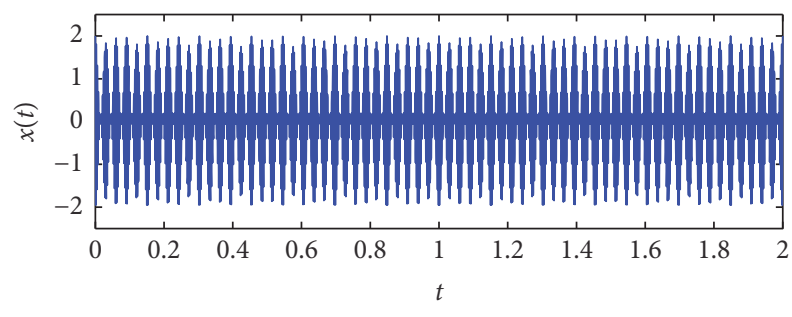

FIGURE 2: The simulated gear vibration signal without noise.

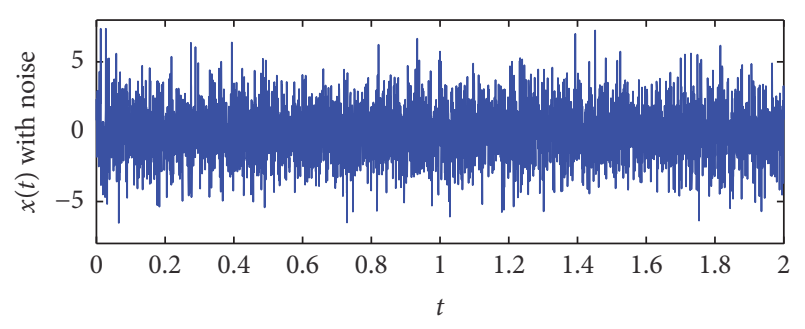

FIGURE 3: The simulated gear vibration signal with noise.

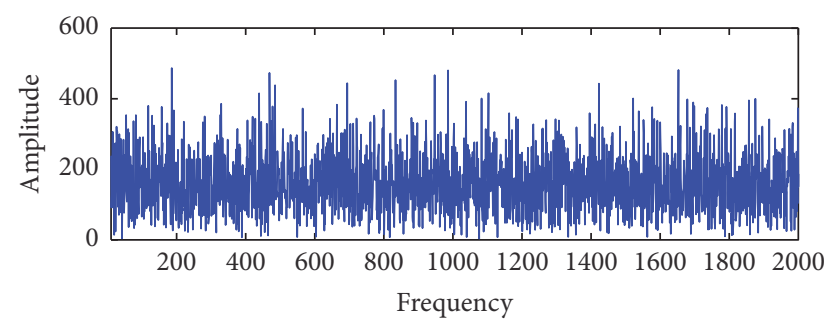

FIgURE 4: The FT of the simulated gear vibration signal with noise.

Now, to obtain the fault features from the simulated gear vibration signal and diagnose the gear faults, the MSCPP and the LCT are applied. According to the above analysis, we first use the MSCPP method to estimate the instantaneous frequency of the simulated gear vibration signal. Because the sampling frequency is $2000 \mathrm{~Hz}$, we let the search range and the search step lengths of the frequency slope be -100 to $100 \mathrm{~Hz} / \mathrm{s}$ and $1 \mathrm{~Hz} / \mathrm{s}$, respectively. The search range and the search step lengths of offset coefficients are 0 to $100 \mathrm{~Hz}$ and $1 \mathrm{~Hz}$, separately. The points of any dyadic time span is 32 . Therefore, we can obtain that $j_{\max }=\log _{2}(N / 32)$, which is equal to 7. Based on these, by applying the MSCPP algorithm, the IF of the simulated gear vibration signal is obtained in Figure 5. In Figure 5, the blue line and the red line represent the actual and estimated instantaneous frequency of the simulated gear vibration signal, respectively. The red line is coincided with the blue line very well in Figure 5, which shows that the MSCPP algorithm can be used to estimate IF of the simulated gear vibration signal efficiently. Based on Figure 6, we can know that the time interval from $1 \mathrm{~s}$ to $1.5 \mathrm{~s}$ can be selected to approach the acceleration process of the gear in a short period of time. Next, based on the gradient of the signal segment from $1 \mathrm{~s}$ to $1.5 \mathrm{~s}$ and the properties of the LCT [30], the optimal LCT spectrum with parameters $(0.93,0.42,0.34,1.89)$ of the selected signal

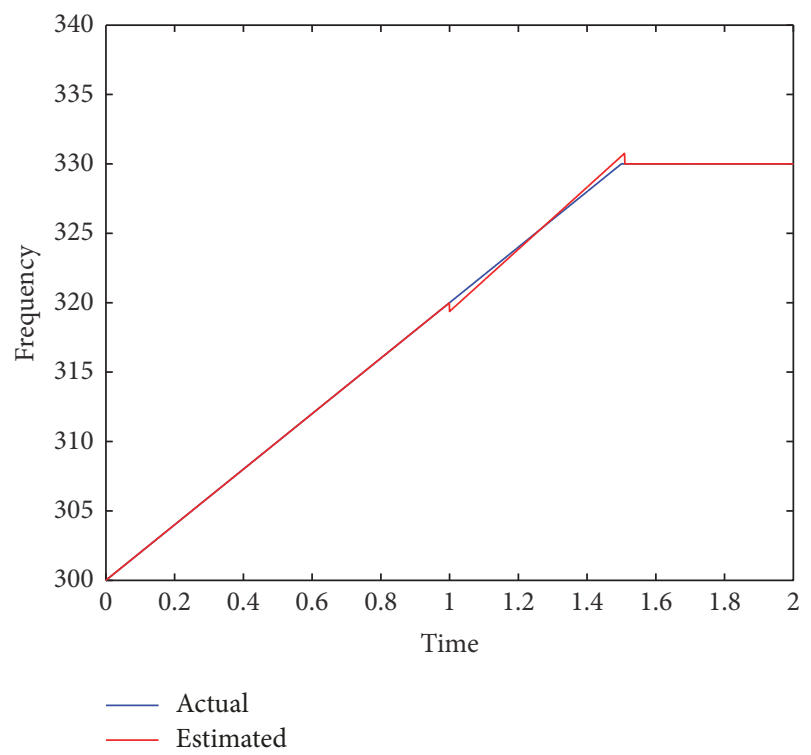

FIGURE 5: Estimated instantaneous frequency of the simulated gear vibration signal.



FIGURE 6: The LCT of the simulated gear vibration signal with noise.

segment is obtained in Figure 6. It is shown that Figure 6 has three peaks, which are $(595,1066),(661,1915)$, and $(727,869.6)$, respectively. The highest peak is $(661,1915)$. The LCT frequency distances of each of the two adjacent peaks are $66 \mathrm{~Hz}$. It is shown that the frequency of $(661,1915)$ is almost ten times of the SRF associated with the LCT. This means that the peaks $(595,1066),(661,1915)$, and $(727,869.6)$ are 9,10 , and 11 times of the SRF associated with the LCT, separately. From the results, we can know that it is very consistent with the analysis of (7).

Moreover, since the results obtained in Figure 6 show that the range of any two adjacent peaks are equal, hence the signal presented in Figure 3 could be considered as the fault gear vibration signal based on the description in $[1,4]$. Thus, the proposed method is an effective method to diagnose the gear fault according to this simulation. 


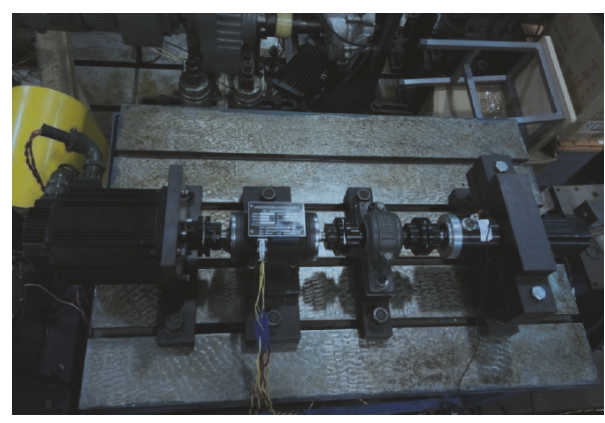

FiguRE 7: Platform of experiment.

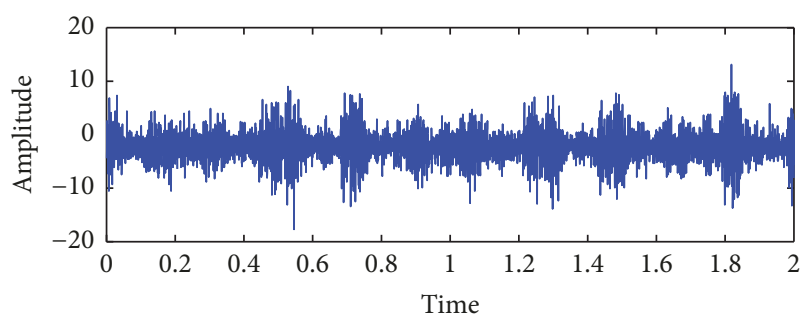

FIGURE 8: The vibration signal obtained from the normal gear.

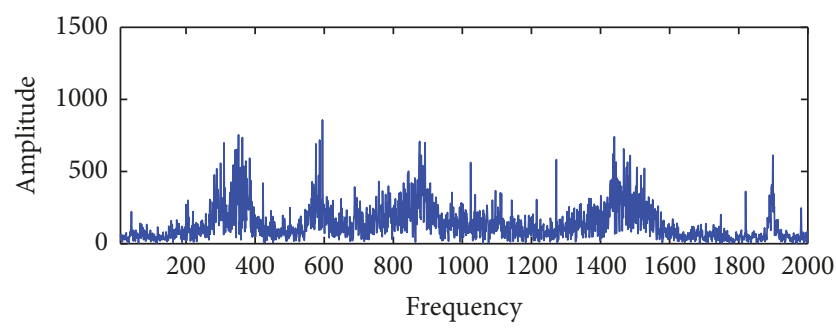

FIGURE 9: The FT of the vibration signal obtained from the normal gear.

\section{Experimental Evaluation}

In Section 3, the validity of the MSCPP and the LCT in gear faults diagnosis has been verified by the signal presented in (7). In order to further verify the correctness of the proposed algorithm, in this part, the gear faults of experiment setup presented in Figure 7 have been used. In this gear experiment equipment, the output gear has 75 teeth and the input pinion gear has 55 teeth. The sample frequency and the sample time of the signals obtained from this experiment equipment are $4000 \mathrm{~Hz}$ and $2 \mathrm{~s}$, respectively.

Firstly, the vibration signal attained from a normal gear in variable speed condition is presented in Figure 8. The signal presented in Figure 8 is nonstationary since the gear signals with low SNR are obtained from variable speed condition. On this account, Figure 9 depicts the FT of the signal shown in Figure 8 which has spectrum aliasing, and it cannot be used to diagnose the gear fault. Next, by applying the method we presented in this paper, we used the MSCPP method to estimate the instantaneous frequency of the gear vibration signal at first. In the MSCPP method, the seeking

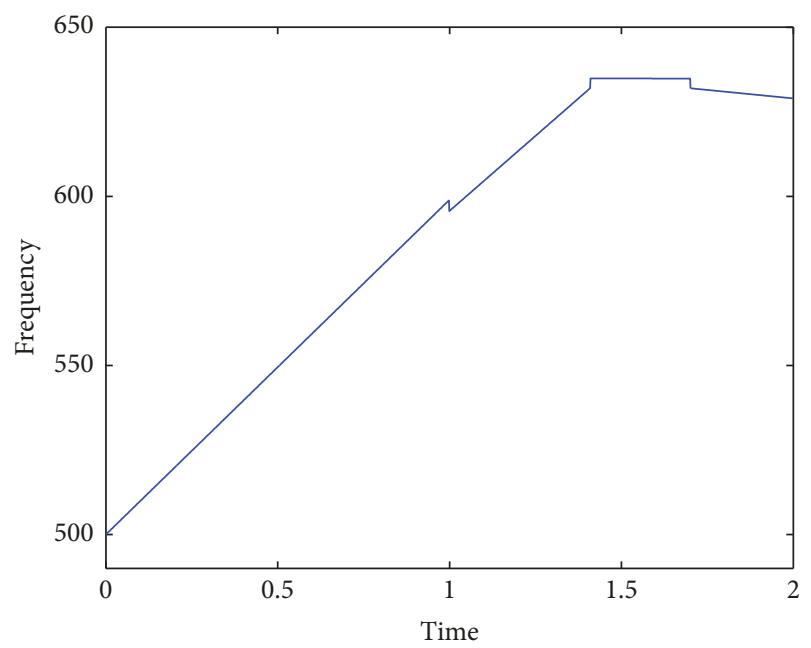

FIGURE 10: Estimated instantaneous frequency of the signal presented in Figure 13.

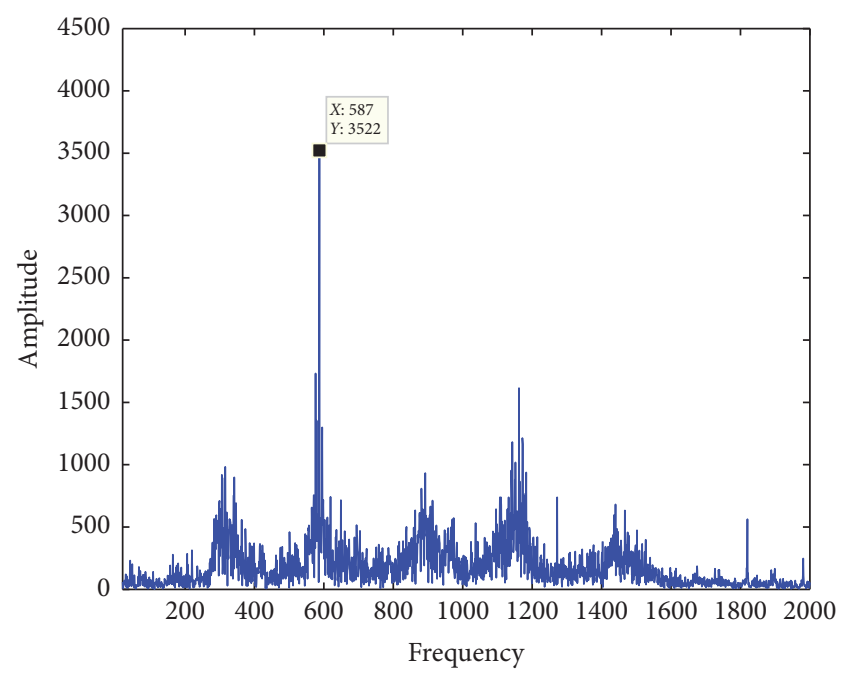

FIGURE 11: The LCT of the selected signal segment.

scope of frequency offset coefficients and slope coefficient are $[0,2000 \mathrm{~Hz}]$ and $[-100,100 \mathrm{~Hz} / \mathrm{s}]$, respectively. The search resolutions of these are chosen as $1 \mathrm{~Hz} / \mathrm{s}$ and $1 \mathrm{~Hz}$, separately. The samples are 4096. Based on these, we can obtain the maximum scales of 7 . Thus, the IF of the gear vibration signal is obtained in Figure 10. From Figure 10, it is easy to know that the time interval from $1 \mathrm{~s}$ to $1.4 \mathrm{~s}$ can be selected to approach the acceleration process of the gear in a short period of time. Subsequently, by utilizing the advantages of the LCT and the properties of the selected signal segment, the optimal LCT spectrum with parameters $(-0.17,1.53,-0.98,2.93)$ of the selected signal segment is shown in Figure 11. We can easily know that Figure 11 has only one peak. That is to say, the signal presented in Figure 8 has not amplitude modulated. Hence, the gear can be seen as a normal gear.

Then, Figure 12 shows a gear vibration signal, which is attained from the gear which has a broken tooth in variable speed condition. The FT of the signal presented 


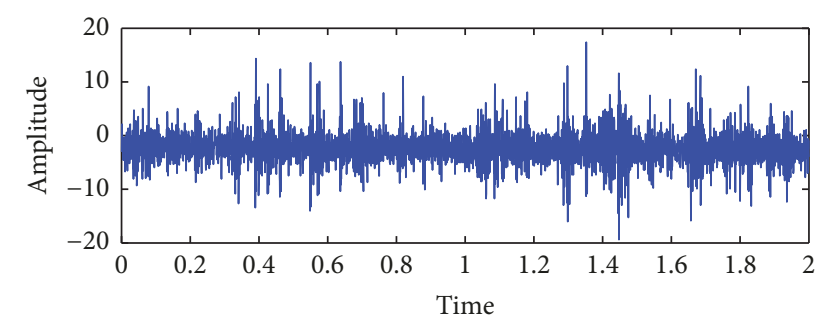

FIGURE 12: The vibration signal obtained from the fault gear.

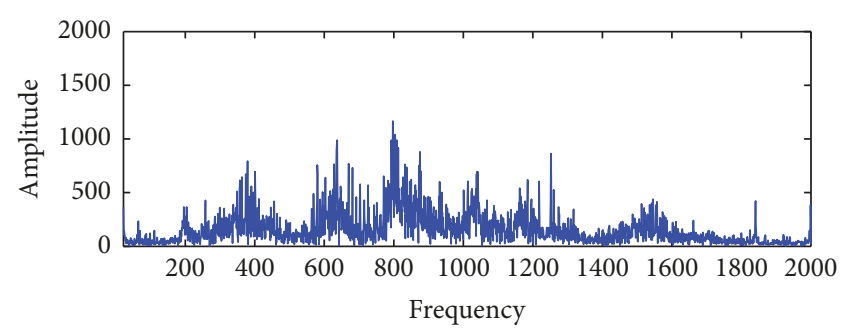

Figure 13: The FT of the vibration signal obtained from the fault gear.

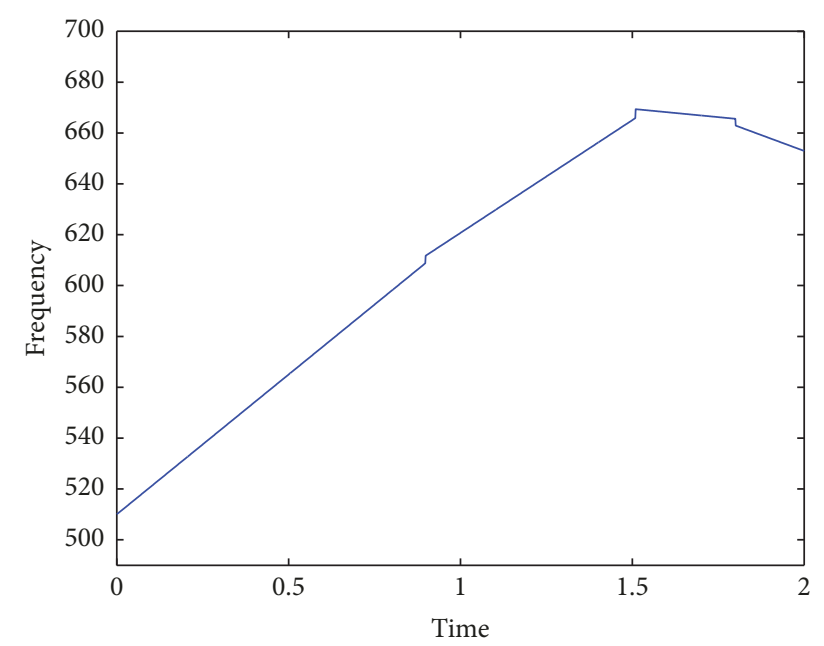

FIGURE 14: Estimated instantaneous frequency of the signal presented in Figure 17.

in Figure 12 is presented in Figure 13, which shows that it also has spectrum aliasing and cannot be used to diagnose the gear fault. Now, by applying the proposed method, the search range of frequency offset coefficients and slope coefficient are also set as $[0,2000 \mathrm{~Hz}]$ and $[-100,100 \mathrm{~Hz} / \mathrm{s}]$. The search resolutions of these are also chosen as $1 \mathrm{~Hz} / \mathrm{s}$ and $1 \mathrm{~Hz}$, separately. The samples are 4096. Based on these, the estimated instantaneous frequency of the gear vibration signal can been obtained in Figure 14 based on the MSCPP method. From Figure 14, the time interval from $1 \mathrm{~s}$ to $1.5 \mathrm{~s}$ can be selected to approach the acceleration process of the gear in a short period of time. Subsequently, according to the above analysis, the optimal LCT spectrum with parameters $(-0.51,0.87,-1.47,0.56)$ of the selected gear vibration signal segment can be obtained in Figure 15. We can know that

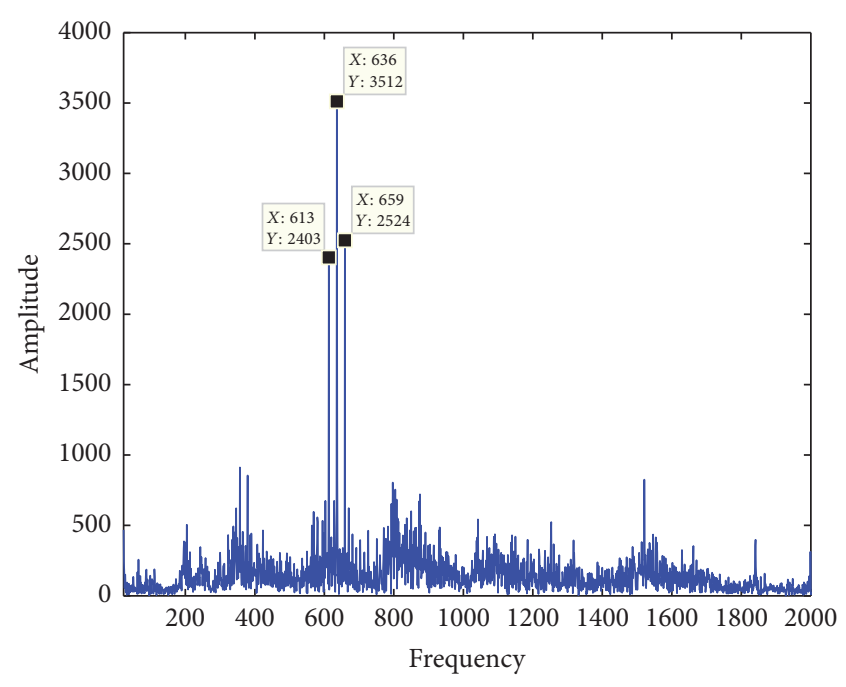

FIGURE 15: The LCT of the selected signal segment from the fault gear.

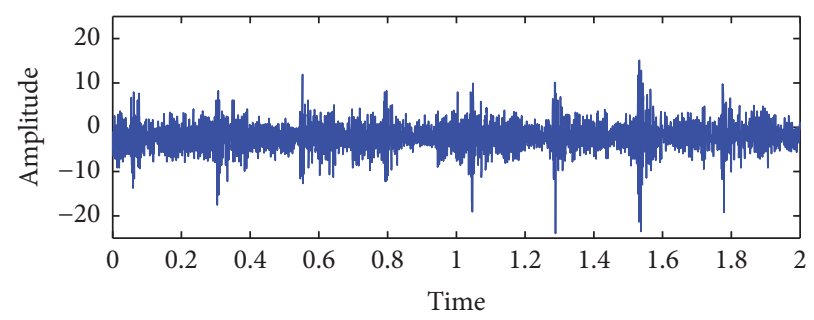

FIGURE 16: Another vibration signal obtained from the fault gear.

Figure 15 has three peaks, that is, $(613,2403),(636,3512)$, and $(659,2524)$. Since $(636,3512)$ is the highest and there are 55 teeth in the pinion gear, thus the input SRF associated with the LCT can be expressed as $636 \mathrm{~Hz} / 55=11.56 \mathrm{~Hz}$ [4], which is equal to half of the frequency distance. That is to say, the meshing frequency is twofold of the SRF. Based on the above analysis, the gear should be considered to have fault happened.

In addition, in order to further verify the correctness of the proposed algorithm, Figure 16 presents another gear vibration signal, which is attained from the fault gear in different variable speed condition. The FT of the signal presented in Figure 16 is presented in Figure 17, which also shows that it cannot be used to diagnose the gear fault. Now, by applying the proposed method, similar to the above cases, the largest scale is also 7. Then, the estimated IF of the gear vibration signal has been obtained in Figure 18 based on the MSCPP method. From Figure 18, the time interval from $1 \mathrm{~s}$ to $1.4 \mathrm{~s}$ can be selected to approach the deceleration process of the gear in a short period of time. Then, the optimal LCT spectrum with parameters $(1.53,0.57,0.42,0.81)$ of the selected gear vibration signal segment can be obtained in Figure 19. It is shown that Figure 15 also has three peaks $(591,2484),(613,3543)$, and $(635,2103)$. Since $(613,3543)$ is the highest and there are 55 teeth in the pinion gear, thus the input SRF associated with the LCT can be expressed as 


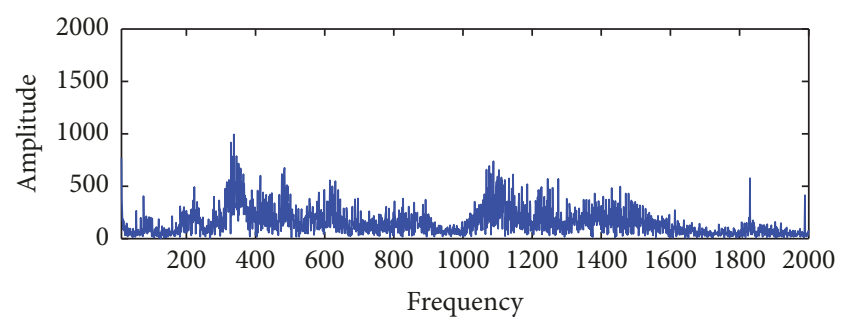

FIGURE 17: The FT of the vibration signal presented in Figure 16.

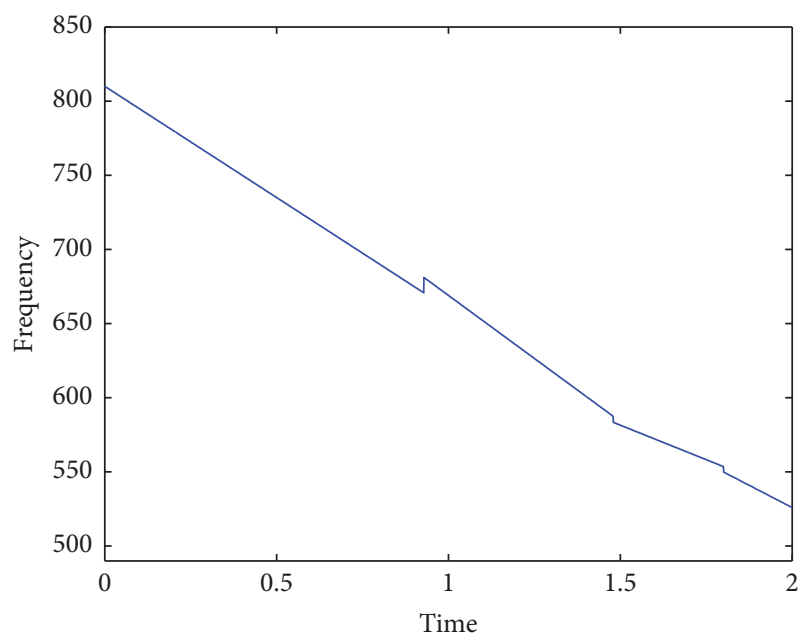

FIGURE 18: Estimated instantaneous frequency of the signal presented in Figure 16.

$613 \mathrm{~Hz} / 55=11.14 \mathrm{~Hz}$. That is to say, the meshing frequency is twofold of the SRF in the LCT domain. Thus, it can be said that the gear also have fault that occurred.

Based on the normal case and two fault cases, it is shown that the method proposed in this can diagnose the gear faults in variable speed condition. However, the MSCPP and LCT method only can show that the gear faults happened, and our research directions will be the diagnosis of the types and the severity of the gear faults.

\section{Conclusion}

In this paper, a method based on the MSCPP and the LCT has been applied to diagnose the gear faults in the variable speed condition for the first time. Firstly, the preliminaries of the MSCPP and the LCT have been presented. Then, the proposed method of the simulated gear vibration signals have been showed. At last, in order to further verify the correctness of the proposed algorithm, the diagnosis of actual gear vibration signals also has been presented. It is indicated that the proposed method can diagnose the gear faults availably. In the future, the diagnosis of early gear faults, intermittent gear faults, and multiple gear faults also will be our future research directions.

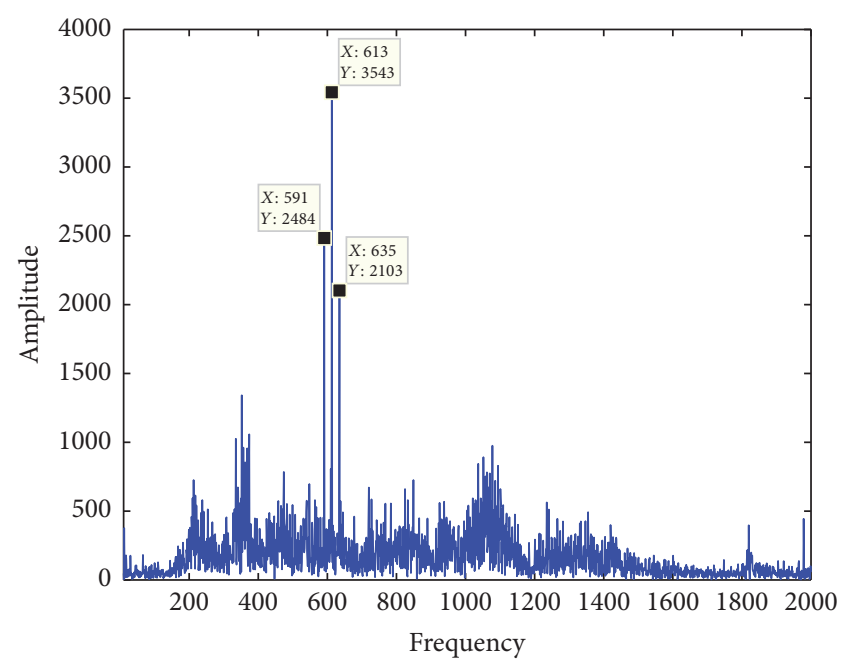

FIGURE 19: The LCT of the selected signal segment presented in Figure 16.

\section{Conflicts of Interest}

The authors declare that they have no conflicts of interest.

\section{Acknowledgments}

This work was supported by the National Natural Science Foundation of China (61374135, 61633005, 61673076, and 51637004), the National Key Research and Development Plan: Important Scientific Instruments and Equipment Development (2016YFF0102200), and Central Military Equipment Development Department Pre-Research Project (41402040301).

\section{References}

[1] P. D. McFadden, "Detecting fatigue cracks in gears by amplitude and phase demodulation of the meshing vibration," Journal of Vibration, Acoustics, Stress, and Reliability in Design, vol. 108, no. 2, pp. 165-170, 1986.

[2] X. Fan and M. J. Zuo, "Gearbox fault detection using Hilbert and wavelet packet transform," Mechanical Systems and Signal Processing, vol. 20, no. 4, pp. 966-982, 2006.

[3] J. Luo, D. Yu, and M. Liang, "Application of multi-scale chirplet path pursuit and fractional Fourier transform for gear fault detection in speed up and speed-down processes," Journal of Sound and Vibration, vol. 331, no. 22, pp. 4971-4986, 2012.

[4] P. Girdhar and C. Scheffer, Practical Machinery Vibration Analysis and Predictive Maintenance, vol. 46, Elsevier, 11 edition, 2004.

[5] Y. Yang, Y. He, J. Cheng, and D. Yu, "A gear fault diagnosis using Hilbert spectrum based on MODWPT and a comparison with EMD approach," Measurement, vol. 42, no. 4, pp. 542-551, 2009.

[6] M. Feldman, "Hilbert transform in vibration analysis," Mechanical Systems and Signal Processing, vol. 25, no. 3, pp. 735-802, 2011.

[7] J. He, S. Yang, and C. Gan, "Unsupervised fault diagnosis of a gear transmission chain using a deep belief network," Sensors, vol. 17, no. 7, article no. 1564, 2017. 
[8] P. Maragos, J. F. Kaiser, and T. F. Quatieri, "Energy separation in signal modulations with application to speech analysis," IEEE Transactions on Signal Processing, vol. 41, no. 10, pp. 3024-3051, 1993.

[9] H. Li, J. Zhao, X. Zhang, and H. Teng, "Gear fault diagnosis and damage level identification based on Hilbert transform and Euclidean distance technique," Journal of Vibro Engineering, vol. 16, no. 8, pp. 4137-4151, 2014.

[10] M. Liang and I. Soltani Bozchalooi, "An energy operator approach to joint application of amplitude and frequencydemodulations for bearing fault detection," Mechanical Systems and Signal Processing, vol. 24, no. 5, pp. 1473-1494, 2010.

[11] J. Mei, J. Jia, R. Zeng, B. Zhou, and H. Zhao, "A multi-order FRFT self-adaptive filter based on segmental frequency fitting and early fault diagnosis in gears," Measurement, vol. 91, pp. 532540, 2016.

[12] P. F. Odgaard and J. Stoustrup, "Gear-box fault detection using time-frequency based methods," Annual Reviews in Control, vol. 40, pp. 50-58, 2015.

[13] V. Sharma and A. Parey, "Gear crack detection using modified TSA and proposed fault indicators for fluctuating speed conditions," Measurement, vol. 90, pp. 560-575, 2016.

[14] X. Tang, Y. Guo, Y. Ding, and H. Zheng, "Application of rolling element bearing envelope analysis based on short time Fourier transition and independent components analysis," Journal of Mechanical Strength, vol. 34, no. 2, pp. 1-5, 2012.

[15] L. Shi, Y. Zhang, and W. Mi, "Application of Wigner-Villedistribution-based spectral kurtosis algorithm to fault diagnosis of rolling bearing," Journal of Vibration Measurement \& Diagnosis, vol. 31, no. 1, pp. 27-31, 2011.

[16] D. Song, C. Lu, and J. Ma, "Gearbox fault diagnosis based on VMD-MSE and adaboost classifier," Vibroengineering Procedia, vol. 14, pp. 120-125, 2017.

[17] C. W. A. W. Fanlei, "Gear fault diagnosis based on LCD and LME demodulation approach," China Mechanical Engineering, vol. 27 , no. 24 , p. 3332.

[18] H. Yuan and C. Lu, "Rolling bearing fault diagnosis under fluctuant conditions based on compressed sensing," Structural Control and Health Monitoring, vol. 24, no. 5, Article ID e1918, 2017.

[19] G. Cheng, X. Chen, H. Li, P. Li, and H. Liu, "Study on planetary gear fault diagnosis based on entropy feature fusion of ensemble empirical mode decomposition," Measurement, vol. 91, pp. 140154, 2016.

[20] F.-C. Zhou, G.-J. Tang, and Y.-L. He, "An Effective Gear Fault Diagnosis Method Based on Singular Value Decomposition and Frequency Slice Wavelet Transform," International Journal of Rotating Machinery, vol. 2016, Article ID 7458956, 8 pages, 2016.

[21] A. Krishnakumari, A. Elayaperumal, M. Saravanan, and C. Arvindan, "Fault diagnostics of spur gear using decision tree and fuzzy classifier," The International Journal of Advanced Manufacturing Technology, vol. 89, no. 9-12, pp. 3487-3494, 2017.

[22] Z. Xing, J. Qu, Y. Chai, Q. Tang, and Y. Zhou, "Gear fault diagnosis under variable conditions with intrinsic time-scale decomposition-singular value decomposition and support vector machine," Journal of Mechanical Science and Technology, vol. 31, no. 2, pp. 545-553, 2017.

[23] P. V. Kane and A. B. Andhare, "Application of psychoacoustics for gear fault diagnosis using artificial neural network," Journal of Low Frequency Noise, Vibration and Active Control, vol. 35, no. 3, pp. 207-220, 2016.
[24] H. Wu and C. Z. Song, "Engine ggearbox fault diagnosis using modified Elman neural network and ACO algorithm," Applied Mechanics and Materials, vol. 190-191, pp. 982-986, 2012.

[25] T. Waqar and M. Demetgul, "Thermal analysis MLP neural network based fault diagnosis on worm gears," Measurement, vol. 86, pp. 56-66, 2016.

[26] X. You and W. Zhang, "Fault diagnosis of frequency converter in wind power system based on SOM neural network," in Proceedings of the 2012 International Workshop on Information and Electronics Engineering, IWIEE 2012, pp. 3132-3136, China, March 2012.

[27] W. Zhao, D. Siegel, J. Lee, and L. Su, "An integrated framework of drivetrain degradation assessment and fault localization for offshore wind turbines," International Journal of Prognostics and Health Management, vol. 4, 2, pp. 462-472, 2013.

[28] C. Lu, L. Tao, and H. Fan, "An intelligent approach to machine component health prognostics by utilizing only truncated histories," Mechanical Systems and Signal Processing, vol. 42, no. 1-2, pp. 300-313, 2014.

[29] M. Moshinsky and C. Quesne, "Linear canonical transformations and their unitary representations," Journal of Mathematical Physics, vol. 12, pp. 1772-1780, 1971.

[30] T. Alieva and M. J. Bastiaans, "Properties of the linear canonical integral transformation," Journal of the Optical Society of America A: Optics and Image Science, and Vision, vol. 24, no. 11, pp. 3658-3665, 2007.

[31] Y.-N. Zhang and B.-Z. Li, “ $\phi$-linear canonical analytic signals," Signal Processing, vol. 143, pp. 181-190, 2018.

[32] S. Xu, Y. Chai, Y. Hu, C. Jiang, and Y. Li, "Reconstruction of digital spectrum from periodic nonuniformly sampled signals in offset linear canonical transform domain," Optics Communications, vol. 348, pp. 59-65, 2015.

[33] R. Tao, B.-Z. Li, Y. Wang, and G. Aggrey, "On sampling of bandlimited signals associated with the linear canonical transform," IEEE Transactions on Signal Processing, vol. 56, no. 11, pp. 54545464, 2008.

[34] S. Xu, Y. Chai, and Y. Hu, "Spectral analysis of sampled bandlimited signals in the offset linear canonical transform domain," Circuits, Systems and Signal Processing, vol. 34, no. 12, pp. 39793997, 2015.

[35] X.-N. Xu, B.-Z. Li, and X.-L. Ma, "Instantaneous frequency estimation based on the linear canonical transform," Journal of The Franklin Institute, vol. 349, no. 10, pp. 3185-3193, 2012.

[36] Q. Feng and B.-Z. Li, "Convolution and correlation theorems for the two-dimensional linear canonical transform and its applications," IET Signal Processing, vol. 10, no. 2, pp. 125-132, 2016.

[37] Y. Guo and B.-Z. Li, "Blind image watermarking method based on linear canonical wavelet transform and QR decomposition," IET Image Processing, vol. 10, no. 10, pp. 773-786, 2016.

[38] E. J. Candès, P. R. Charlton, and H. Helgason, "Detecting highly oscillatory signals by chirplet path pursuit," Applied and Computational Harmonic Analysis, vol. 24, no. 1, pp. 14-40, 2008.

[39] J. Luo, S. Zhang, M. Zhong, and Z. Lin, "Order spectrum analysis for bearing fault detection via joint application of synchrosqueezing transform and multiscale chirplet path pursuit," Shock and Vibration, vol. 2016, Article ID 2976389, 11 pages, 2016. 


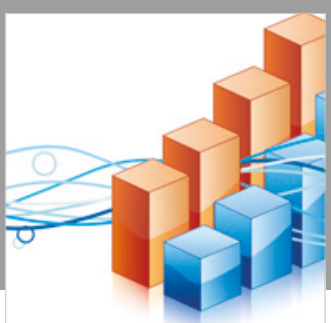

Advances in

Operations Research

\section{-n-m}
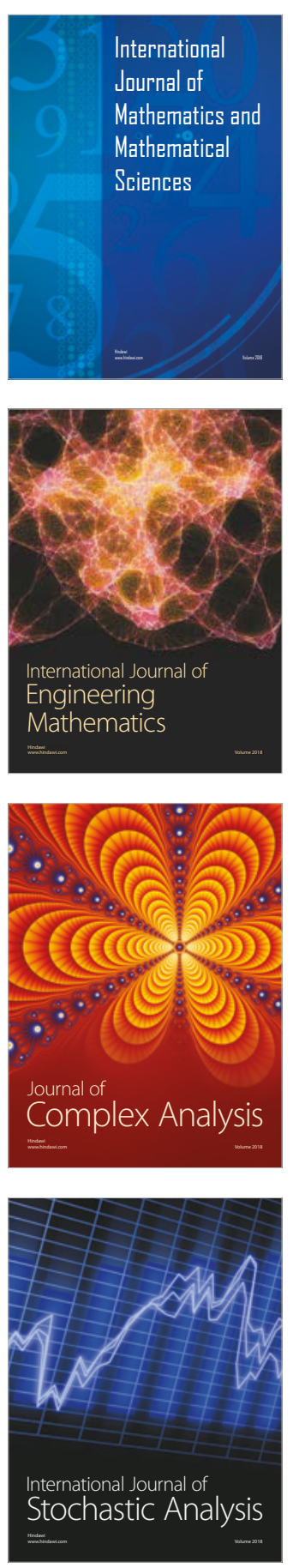
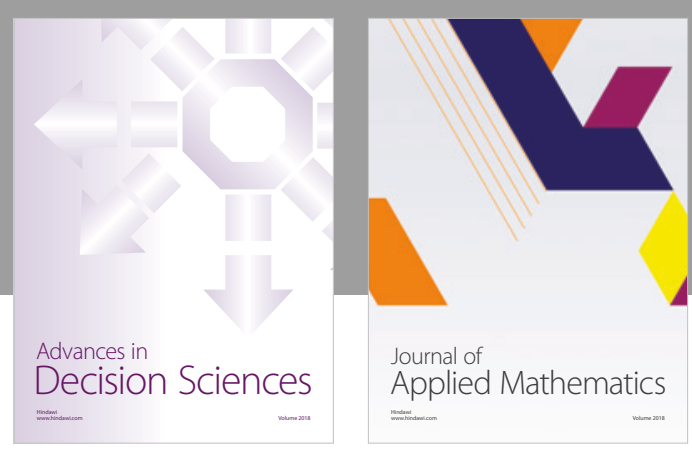

Journal of

Applied Mathematics
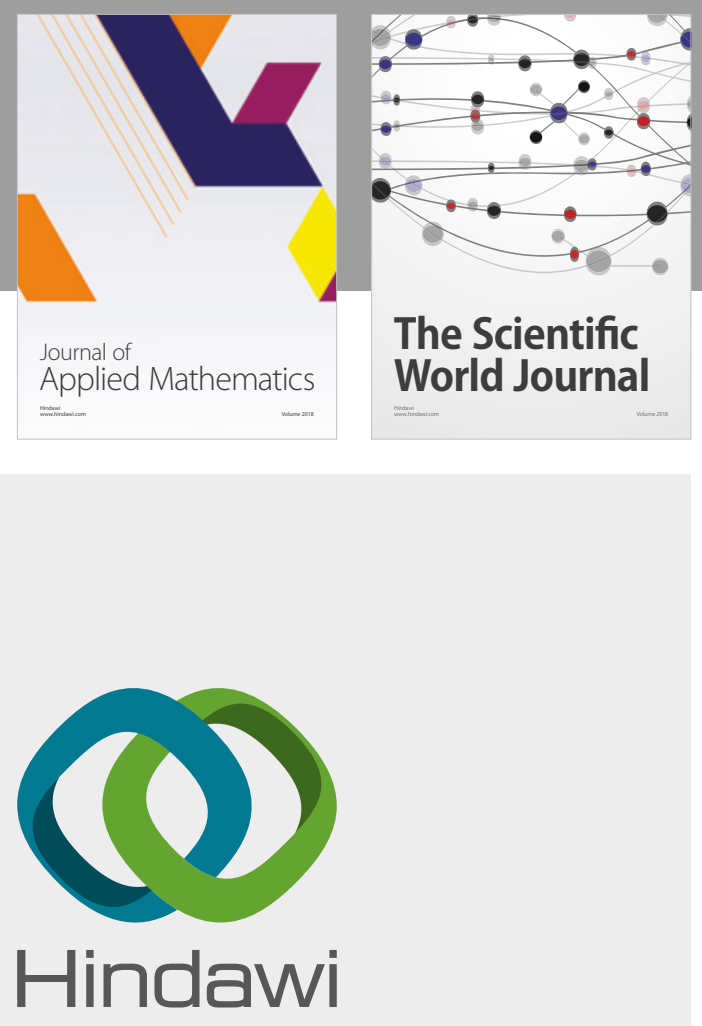

Submit your manuscripts at

www.hindawi.com

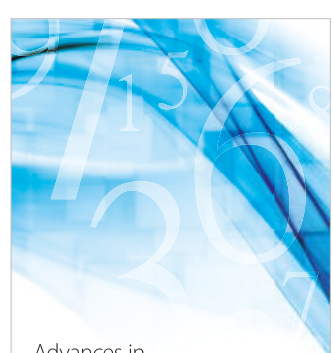

Advances in
Numerical Analysis
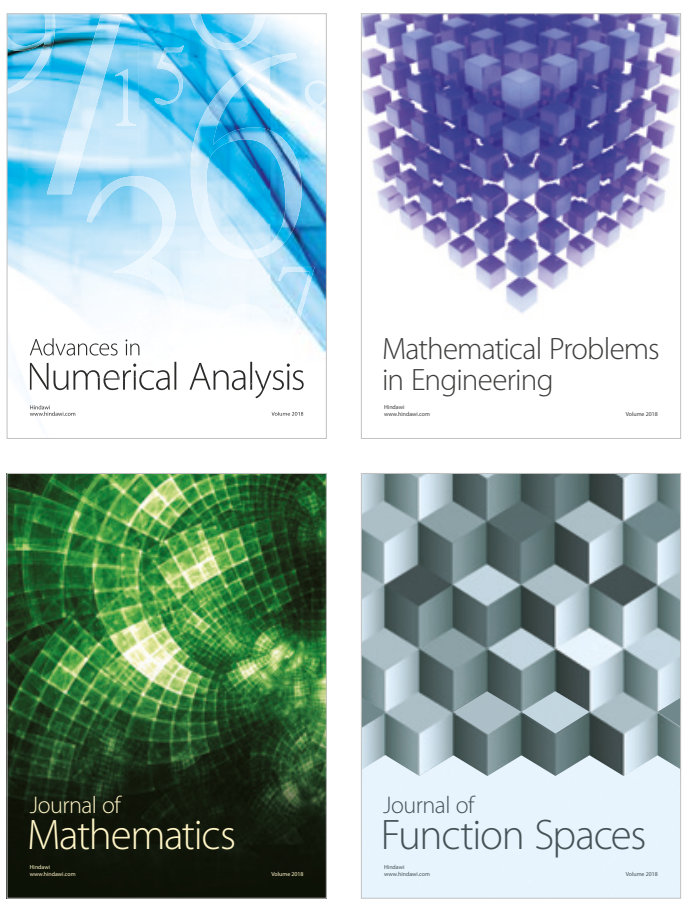

Mathematical Problems in Engineering

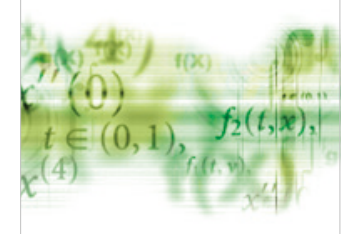

International Journal of

Differential Equations

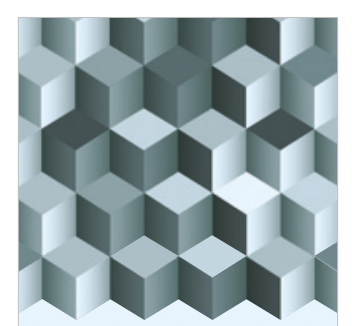

Journal of

Function Spaces
The Scientific

World Journal

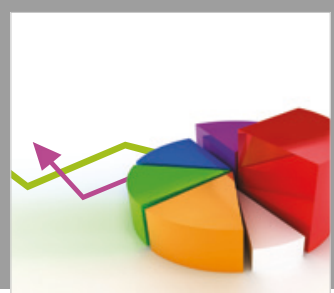

Journal of

Probability and Statistics
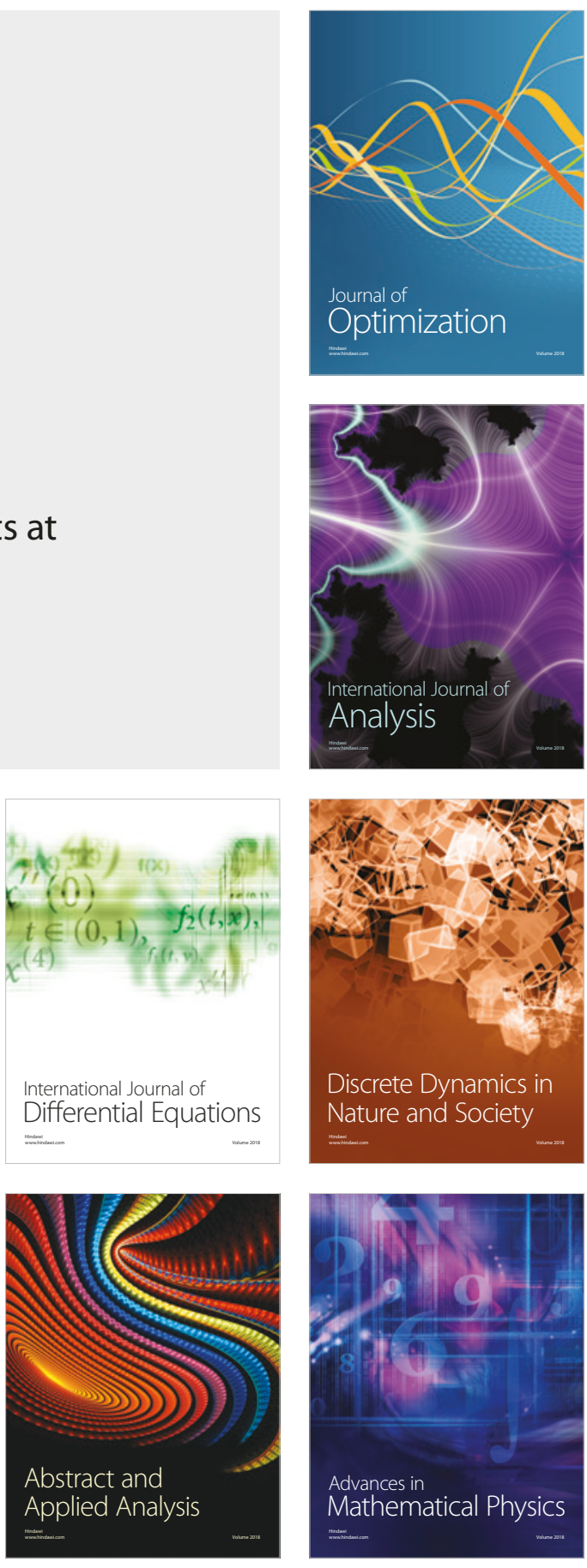